\title{
The effect of mean axial and torsional stresses on the fatigue strength of 34CrNiMo6 high strength steel
}

\author{
Luis Pallarés-Santasmartas ${ }^{1,2}$, Joseba Albizuri ${ }^{2}$, Nelson Leguinagoicoa ${ }^{2}$, Nicolas $_{\text {Saintier }}^{3}$ \\ and Jonathan Merzeau ${ }^{3}$ \\ ${ }^{1}$ Colegio Gaztelueta, Barrio de Artaza, 87, 48940 Lejona, Spain \\ ${ }^{2}$ Departamento de Ingeniería Mecánica, Escuela de Ingeniería de Bilbao, UPV/EHU. Plaza Ingeniero \\ Torres Quevedo, 1, 48013 Bilbao, Spain \\ ${ }^{3}$ École Nationale Supérieure d'Arts et Métiers ParisTech à Bordeaux-Talence. Esplanade des Arts et \\ Métiers - 33405 TALENCE Cedex, France
}

\begin{abstract}
The present study consists of a theoretical, experimental and fractographic investigation of the effect of superimposed static axial and shear stresses on the high cycle fatigue behavior of a 34CrNiMo6 high strength steel in quenched and tempered condition (UTS $=1210 \mathrm{MPa}$ ), commonly employed in highly stressed mechanical components. The Haigh diagrams for the axial and torsional cases under different values of mean stress were obtained. In both cases, experimental results showed that increasing the mean stress gradually reduces the stress amplitude that the material can withstand without failure. The results of the present tests are compared with the theoretical predictions from Findley, based on the maximum damage critical plane; and the methods of Marin and Froustey, which are energetic based criterions. Froustey's method shows the best agreement with experimental results for torsional fatigue with mean shear stresses, showing a non-conservative behaviour for the axial fatigue loading case. Macro-analyses and micro-analyses of specimen fracture appearance were conducted in order to obtain the fracture characteristics for different mean shear stress values under torsion fatigue loading.
\end{abstract}

\section{Introduction}

Modelling the effect of medium stresses is a vitally important issue in fatigue. Not in vain, the influence of the mean stress $\sigma \mathrm{m}$ on the fatigue limit was already observed as one of the main factors influencing the fatigue resistance by Wöhler in 1870 [1], who pointed out that the fatigue limit decreased with the increase of the mean stress $\sigma_{\mathrm{m}}$.

The relative importance of axial mean stresses has been especially noted in Papuga's exhaustive comparison of multi-axial methods in 2011 [2], who concluded that the ability to correctly capture the effect of axial and shear mean stresses is a determining factor in the ability of a multi-axial fatigue method. Although virtually all multiaxial methods take into 
account the influence of axial mean stresses, the way in which they treat the effect of these stresses can differ greatly.

With respect to the mean shear stresses, a widespread hypothesis in the literature is that the mean shear stress does not influence the torsional fatigue limit as long as the maximum shear stress is below the yield limit in shear $\tau_{\mathrm{yp}}$. This theory is based, among others, on Smith's research in the 30 s and 40 s of the last century $[3,4]$.

However, there are many trials in which the effect of mean shear stresses is appreciable even for elastic stresses, such as the experimental campaigns conducted by Sauer [5], Findley [6] or Chodorowski [7]. Figure 1 shows the results obtained by Sauer for a 14S-T aluminium.

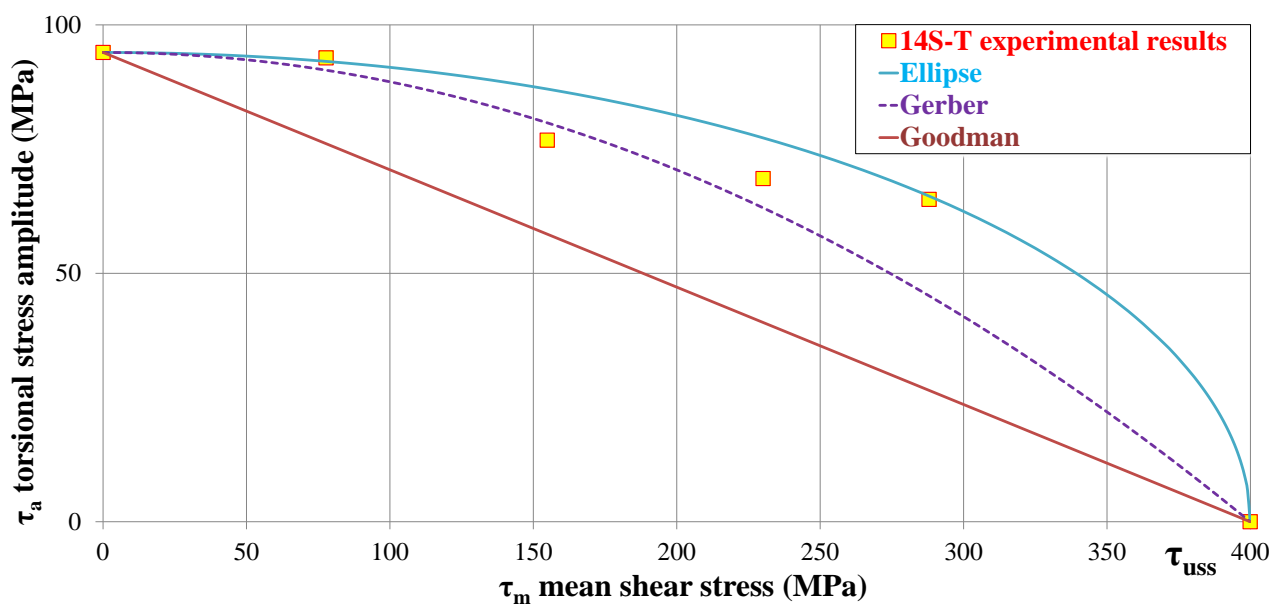

Fig. 1. Torsional Haigh diagram with experimental results for a $14 \mathrm{~S}-\mathrm{T}$ aluminium alloy [5] at $10^{7}$ cycles together with predictions from various empirical lines.

In any case, it can be stated that the influence of mean shear stresses is less than that of mean normal stresses, especially in ductile materials. In this sense, modern design standards such as FKM Guideline [8] take into account the effect of average shear stresses on fatigue. The effect on ductile steels is approximately $58 \%$ of the effect of static axial stresses at the axial fatigue limit. This is expressed through equation (1), which shows the relationship between the slopes of the axial and torsion Haigh diagrams, $M_{\sigma}$ and $M_{\tau}$ respectively:

$$
M_{\tau}=\frac{M_{\sigma}}{\sqrt{3}}
$$

A greater sensitivity to mean axial stresses than torsion was observed by Findley [9], and also in a recent article by Mayer et al. [10] of a spring steel.

In order to investigate the relationship between mean shear and axial stresses, the authors have decided to conduct both a torsional fatigue campaign with mean shear stresses and an axial fatigue campaign with mean axial stresses, and to compare the results with the predictions from some of the best known multiaxial fatigue theories.

\section{STATIC AND FATIGUE TESTS}

The material chosen for the tests was 34CrNiMo6 steel, supplied by Thyssen-Krupp in 30 $\mathrm{mm}$ diameter bars and in the hardened and tempered state. The choice of this material is 
based on its habitual use in parts of high responsibility subjected to fatigue. Its monotonic properties are shown in Table 1. As it can be seen, this is a ductile material despite its high mechanical strength.

Table 1. Static properties of $34 \mathrm{CrNiMo6}$ steel used in the multiaxial campaign

\begin{tabular}{|c|c|c|}
\hline Monotonic properties & Symbol & Value \\
\hline Ultimate Tensile Strength & $\sigma_{\mathrm{UTS}}$ & $1210 \mathrm{MPa}$ \\
\hline Yield strength & $\sigma_{\mathrm{yp}} 0.2$ & $1084 \mathrm{MPa}$ \\
\hline Rupture strain & $\mathrm{A}(\%)$ & $12.18 \%$ \\
\hline Reduction of area & $\mathrm{Z} \mathrm{( \% )}$ & $60.17 \%$ \\
\hline
\end{tabular}

For axial and torsional fatigue tests, as in other similar tests [11], specimens with "hourglass" geometry have been chosen to reduce dispersion by concentrating faults in a localized area of the specimen. The dimensions of the torsional and axial specimens are respectively shown in Figures 2 and 3.

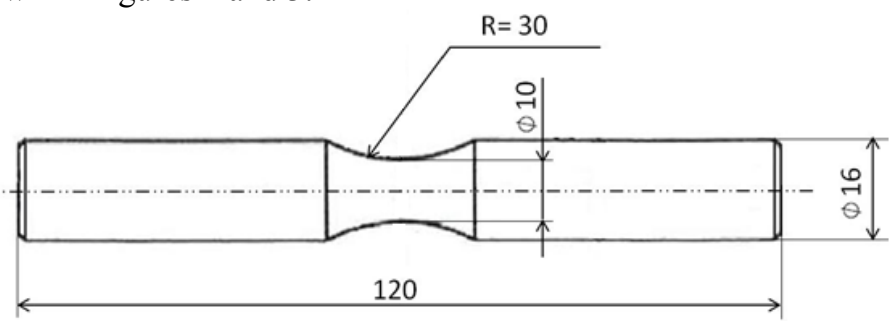

Fig. 2. Hourglass specimen used in the torsional fatigue tests. Dimensions in millimetres.

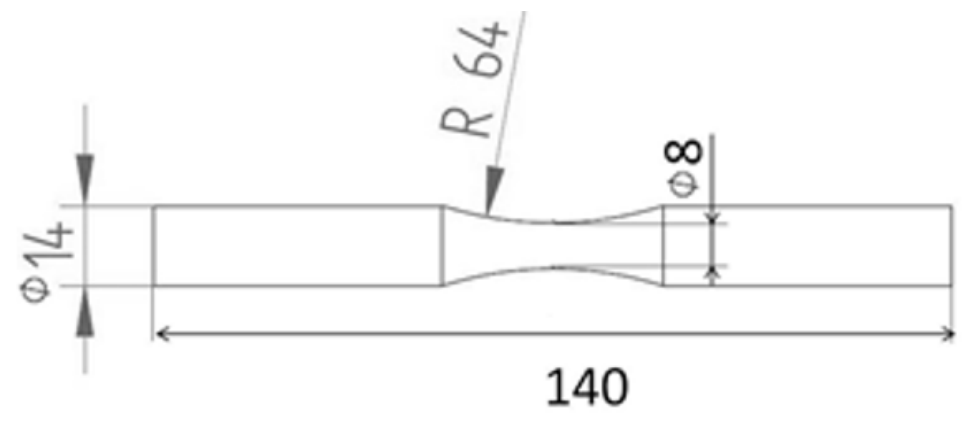

Fig. 3. Hourglass specimen used in the axial fatigue tests. Dimensions in millimetres.

The torsional fatigue tests were carried out in Bordeaux (France) with servo-hydraulic equipment with multiple actuators allowing bending-torsion loads. This machine has previously been used in other experimental campaigns [12], and allows a maximum torque of $150 \mathrm{~N} \cdot \mathrm{m}$ to be applied. To avoid possible bending, the bending actuators were checked at all times and set at $0 \mathrm{~N} \cdot \mathrm{m}$. All tests were performed at $50 \mathrm{~Hz}$.

Figure 4 shows the results for the inclined part of the S-N torsional amplitude curve for different constant mean torsion values. As can be seen, the effect of the mean torsion is clearly measurable in the finite life zone. 


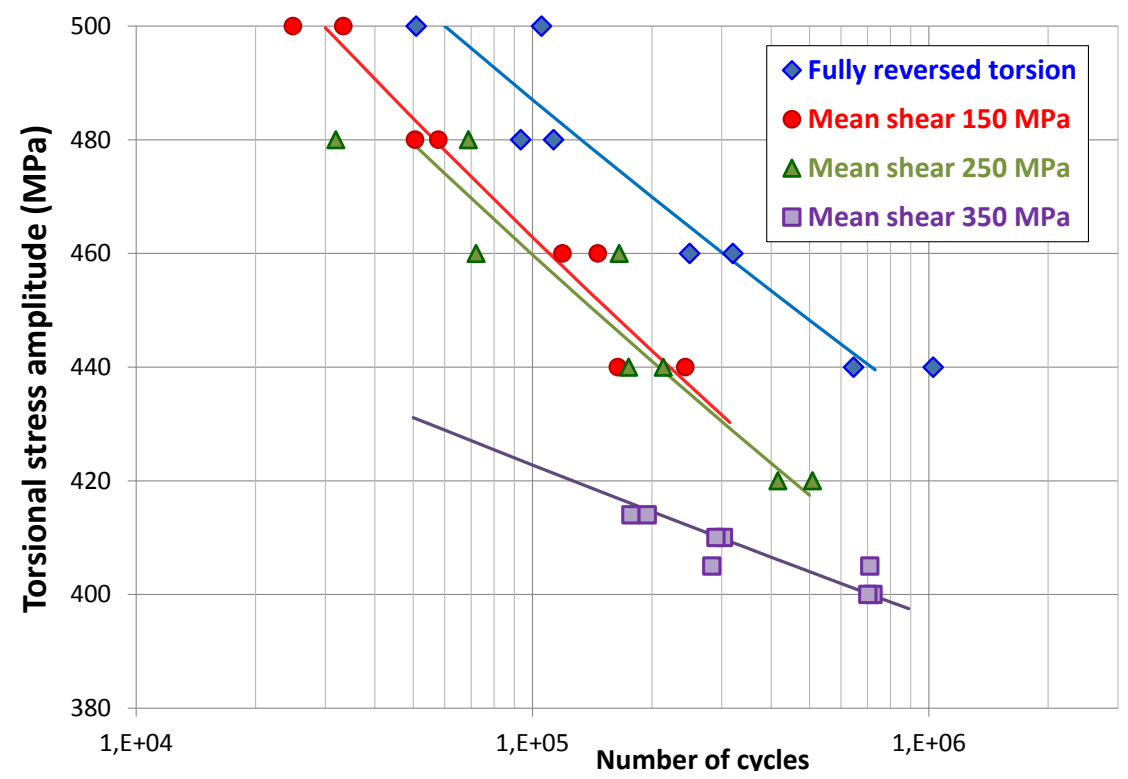

Fig. 4. Cyclic torsional S-N curves for different values of mean shear stress

The uniaxial tests were performed in the Faculty of Engineering of Bilbao on a Instron $8801 \pm 100 \mathrm{KN}$ servohydraulic axial testing machine with $8 \mathrm{~mm}$ diameter specimens following ASTM E 466-15 [13]. Figure 5 shows the axial S-N curves for 4 different stress ratios.

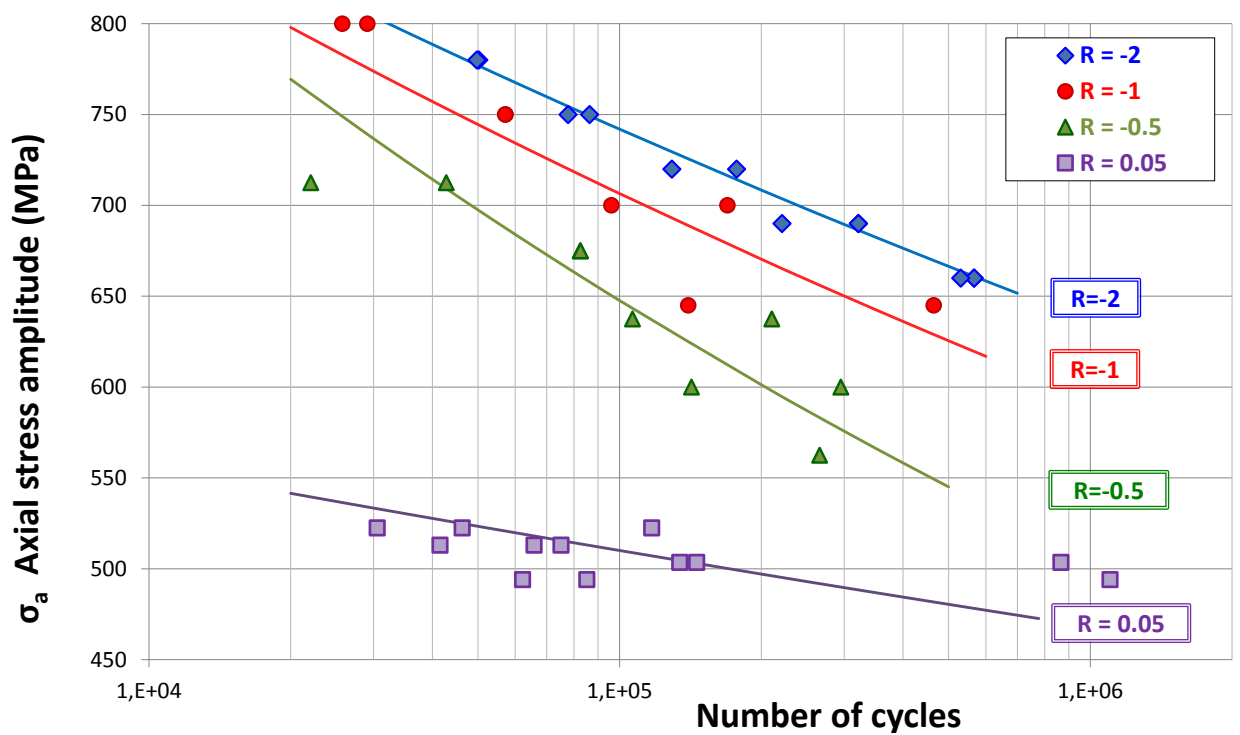

Fig. 5. Axial S-N curves for different stress ratios

The sensitivity to the mean torsional stresses $\mathrm{M}_{\tau}$, defined as the slope of the Haigh diagram in torsion, can be calculated for the case of mean torsion of $350 \mathrm{MPa}$ through equation (2). 


$$
M_{\tau}=\frac{\tau_{a(R=-1)}-\tau_{a\left(\tau_{m}=350\right)}}{350}
$$

Similarly, the sensitivity to axial mean stresses can be calculated as the slope of the axial Haigh diagram, in this case using the data $R=-1$ and $R=0.05$ in equation (3):

$$
M_{\sigma}=\frac{\sigma_{a(R=-1)}-\sigma_{a(R=0.05)}}{\sigma_{m(R=0.05)}}
$$

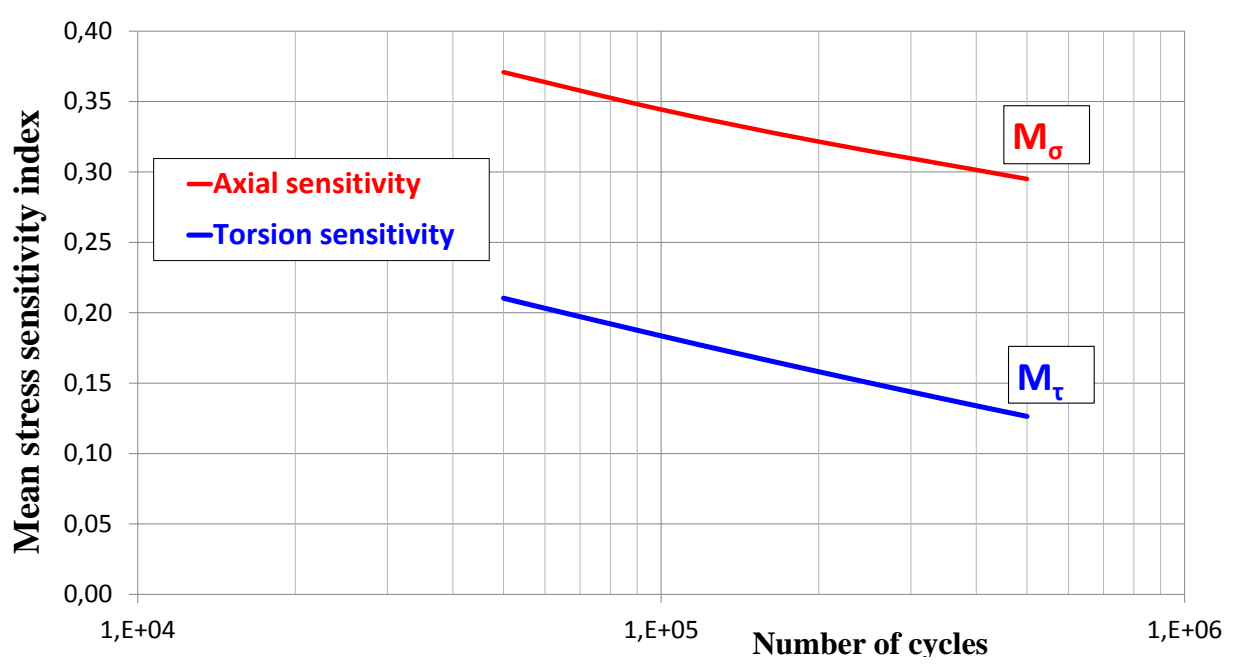

Fig. 6. Axial and mean shear stress sensitivity indices for the range 50,000 $<$ Ncycles $<500,000$

Using equations (2) and (3) a representation of sensitivities to mean axial and shear stresses as a function of the number of cycles can be made, showing the values of M $\sigma$ and $\mathrm{M} \tau$ for the range 50,000< Ncycles $<500,000$ in Figure 6 . As it can be seen, the sensitivity to axial mean stresses is notably higher than for shear mean stresses. In addition, both sensitivities decrease with the number of cycles.

\section{Correlations of experiments with multiaxial fatigue methods}

This paper will analyze the correlation of experimental results obtained with various formulations of multi-axial fatigue whose analytical expressions for cases of uniaxial and torsional fatigue can be obtained in a simple way. Among the methods chosen are the formulations of Findley [14], based on the critical plane method of maximum damage; and the energetic methods of Marin [15], Froustey [16]. Findley's Critical Plane Method [14] was published in 1956 along with experimental fatigue tests on an AISI 4340 steel. This method looks first for the plane in which the damage function (4) is maximized:

$$
\tau_{a}^{*}(\varphi, \theta)+\alpha_{F} \cdot \sigma_{\max } *(\varphi, \theta)
$$

Once the pair of angles has been obtained $\left(\phi^{*}, \theta^{*}\right)$ in which this function is maximum, the criterion is applied by means of the expression (5), where $\alpha_{F}$ and $\lambda_{F}$ are material parameters that can be adjusted with the fully reversed axial and torsional fatigue strengths.

$$
\tau_{a}^{*}\left(\varphi^{*}, \theta^{*}\right)+\alpha_{F} \cdot \sigma_{\max } *\left(\varphi^{*}, \theta^{*}\right) \leq \lambda_{F}
$$


Since it is not necessary for the Findley function plane to be the maximum shear amplitude, there is an influence of the mean shear stresses, which can be represented by the relationship (6):

$$
\tau_{a}=\frac{\sqrt{\lambda_{F}{ }^{2} \cdot \alpha_{F}{ }^{2}+\lambda_{F}{ }^{2}-\alpha_{F}{ }^{2} \tau_{m}{ }^{2}}-\alpha_{F}{ }^{2} \cdot \tau_{m}}{1+\alpha_{F}{ }^{2}}
$$

The application of the Findley method to the uniaxial case leads to equation (7):

$$
\sigma_{a}=2 \cdot \sqrt{\lambda_{F}^{2} \cdot \alpha_{F}^{2}+\lambda_{F}^{2}-\alpha_{F} \cdot \beta_{F} \cdot \sigma_{m}}-2 \cdot \alpha_{F} \cdot \beta_{F}
$$

The constants $\alpha_{\mathrm{F}}$ and $\lambda_{\mathrm{F}}$ depend on the value of the fatigue ratio $\kappa=\sigma-1 / \tau-1$. When $\kappa$ takes a value of 2 , which corresponds to Tresca's criterion, $\alpha_{\mathrm{F}}$ takes the value of 0 , therefore there is no effect of the average voltages. For materials that follow the Rankine criterion of maximum main stress, i.e.: $\kappa \approx 1$, the influence of medium stresses is very high.

Marin's method [15] was presented at the first International Fatigue Congress in 1956 along with an extensive database compiled by the author, consisting of 289 fatigue limits from 23 test series. This method can be generally expressed by equation (8). Its physical interpretation is a balance of the distortion energy between the uniaxial static case and the fully reversed uniaxial fatigue strength.

$$
\left(\frac{\sqrt{3} \cdot \sqrt{J_{2, a}}}{\sigma_{-1}}\right)^{2}+\left(\frac{\sqrt{3} \cdot \sqrt{J_{2, m}}}{\sigma_{U T S}}\right)^{2} \leq 1
$$

This method requires only fully reversed axial fatigue strength $\sigma_{-1}$ and ultimate tensile strength $\sigma_{U T S}$. For the particular case of pure torsion it can be defined by the expression (9), which is an ellipse in the torsional Haigh diagram.

$$
\tau_{a} \leq \frac{\sigma_{-1}}{\sqrt{3}} \cdot \sqrt{1-\left(\frac{\tau_{m}}{\sigma_{u t s} / \sqrt{3}}\right)^{2}}
$$

As can be seen after examining equation (9), Marin's method uses the Von Mises ratio, and therefore the ratio between the fully reversed axial fatigue limits $\left(\sigma_{-1}\right)$ and the fully reversed torsional fatigue limit $\left(\tau_{-1}\right)$ is assumed to be equal to $\sqrt{3}$. For axial fatigue case, the application of the method leads to the elliptical relationship, equation (10):

$$
\sigma_{a} \leq \sigma_{-1} \cdot \sqrt{1-\left(\frac{\sigma_{m}}{\sigma_{u t s}}\right)^{2}}
$$

Another widely used method is that of Froustey [16], which is based on a balance of the total deformation energy. The application of this method to the case of torsional fatigue with mean shear stresses leads to the analytical formula of equation (11):

$$
\tau_{a} \leq \frac{\sigma_{-1}}{\kappa} \cdot \sqrt{1-\left(\frac{\tau_{m}}{\sigma_{u t s} / \kappa}\right)^{2}}
$$


Which, like Marin's method, is also an ellipse in the torsional Haigh diagram, but using the experimental relationship $\kappa=\sigma_{-1} / \tau_{-1}$ instead of the theoretical relationship of Von Mises. The application of the Froustey method to the case of axial fatigue with mean axial stresses leads to the same elliptical relationship as Marin's method, equation (10), which is not in agreement with the experimental results of ductile materials, as shown in [17].

Figures 7 and 8 show the Haigh torsion and axial diagrams respectively together with the experimental results and theories analyzed for $5 \cdot 10^{5}$ cycles.

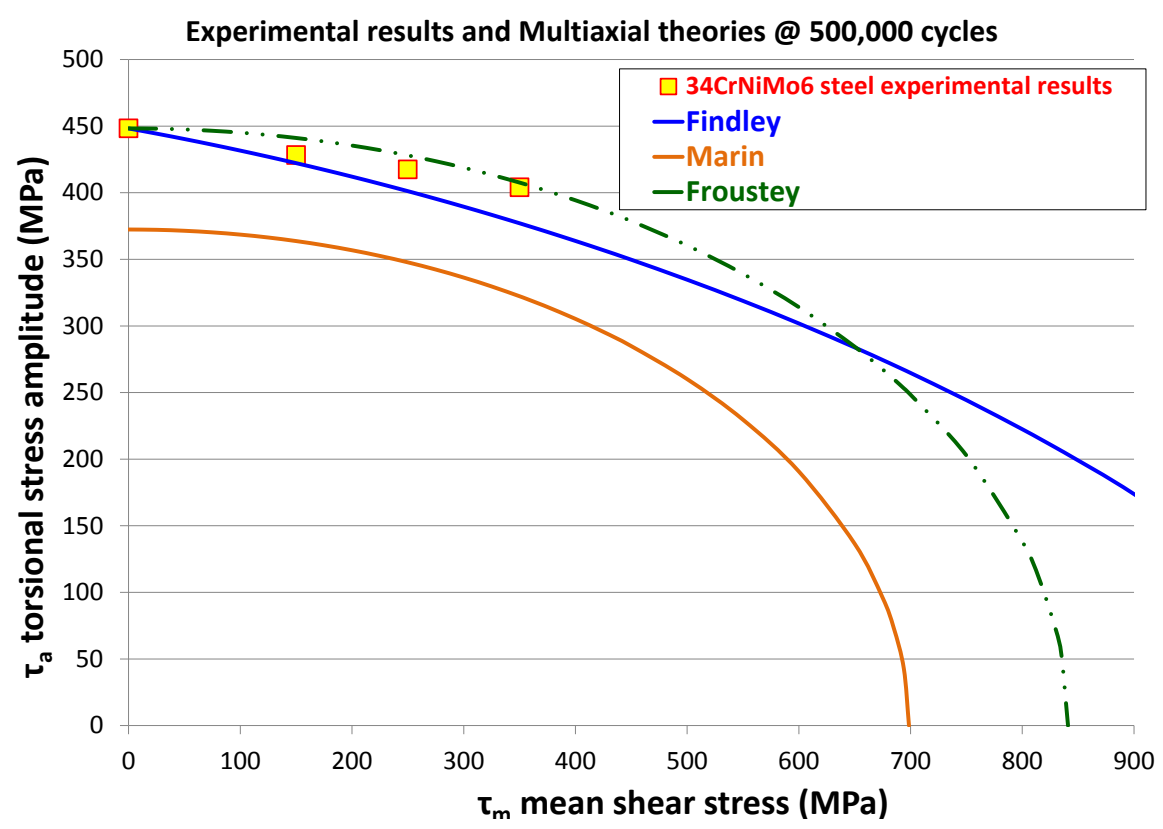

Fig. 7. Torsional Haigh diagram with experimental results and theories for $\mathrm{N}=5-10^{5}$ cycles

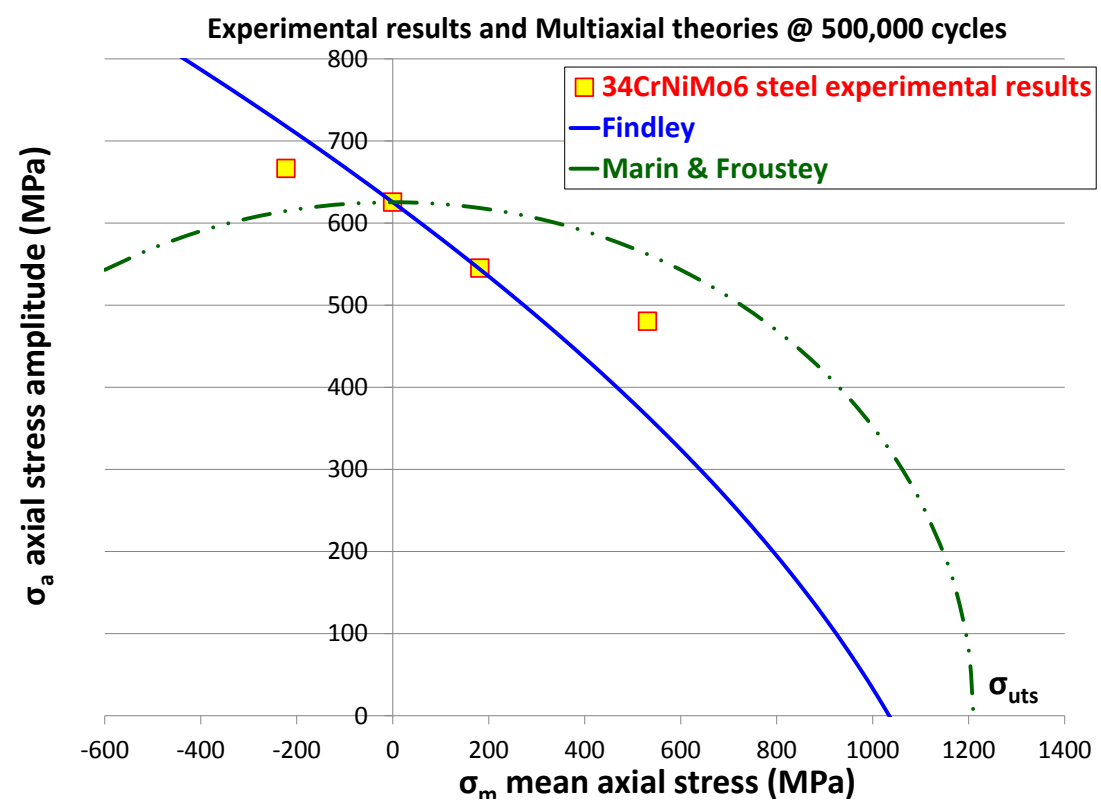

Fig. 8. Axial Haigh diagram with experimental results and theories for $\mathrm{N}=5-10^{5}$ cycles 
As can be seen, the Froustey method obtains a good correlation with the experimental results in torsion, being slightly conservative. As for its axial behaviour, it is extremely non-preservative, foreseeing a more ductile behaviour than that observed in this steel. The Marin method shows an extremely conservative behaviour for this steel in the case of torsional loads due to the use of the Von Mises ratio. On the other hand, in uniaxial fatigue, like the Froustey method, it is not conservative for mean tension stresses, and conservative for the mean compression loading case. Findley's critical plane methodology is shown to be slightly conservative in torsional fatigue for this steel, and with a higher degree of conservatism for axial fatigue, except for the mean compression loading case.

\section{Fractographic analysis}

In general, there is similar behaviour to that observed by other researchers when the number of cycles is relatively high [11]: in tests with failures in a high number of cycles, a visible crack was not observed until a few cycles before rupture. In the case of axial fatigue, in some specimens the failure started inside the specimen. However, in the case of torsion, the crack always started and propagated on the surface.

In torsion, the nucleation of the cracks took place in planes close to the plane of maximum shear amplitude, independently of the applied shear stress, with maximum angular deviations of $15^{\circ}$ (Figures 9 and 10 ).

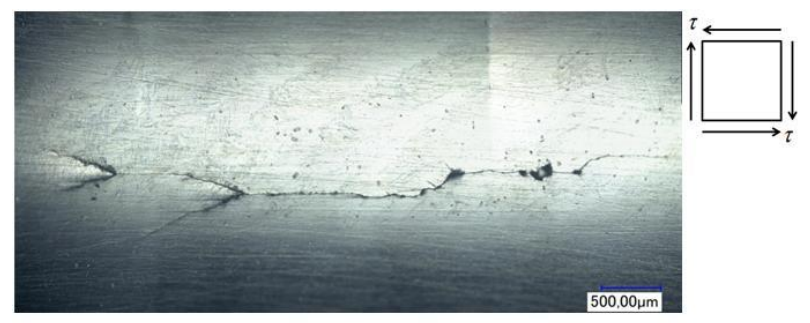

Fig. 9. Fractured surface for $\tau_{\mathrm{a}}=480 \mathrm{MPa} ; \tau_{\mathrm{m}}=0 \mathrm{MPa}$; Ncycles $=93,400$

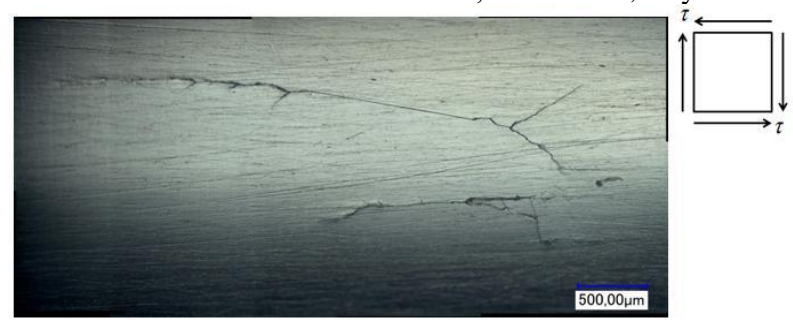

Fig. 10. Fractured surface for $\tau_{\mathrm{a}}=480 \mathrm{MPa} ; \tau_{\mathrm{m}}=250 \mathrm{MPa}$; Ncycles $=68,920$

\section{Conclusions}

The tests show a greater sensitivity to mean axial stresses than to shear stresses for the $34 \mathrm{CrNiMo6}$ steel studied, for a range of number of cycles $5 \cdot 10^{4}<\mathrm{N}<5 \cdot 10^{5}$. Among the multi-axial fatigue methods analyzed, the Froustey method obtains an excellent correspondence in torsion but, following the elliptical relation in axial, it shows a nonconservative behaviour for tensile mean loading. The Marin method, which assumes the von Mises ratio for axial and torsional fatigue limits, is highly conservative in torsion and highly non-preservative in axial. Findley's Critical Plane methodology shows a generally conservative behaviour for this steel, except for mean compression stresses in axial fatigue. 
The authors wish to acknowledge the financial support received from the Department of Research and Development of the Basque Government, through the Project Reference: Grupos GV IT947-16

\section{References}

1. A. Wöhler. On strength tests with iron and steel. Zeitschrift für Bauwesen, $\mathbf{X X}, 73-106$ (1870).

2. J. Papuga. A survey on evaluating the fatigue limit under multiaxial loading. Int. J. Fatigue, 33:153-165 (2011)

3. J.O. Smith. The effect of range of stress on the torsional fatigue strength of steel. University of Illinois Engineering Experiment Station, Bulletin series No. 316 (1939)

4. J.O. Smith. The effect of range of stress on the fatigue strength of metals. University of Illinois Engineering Experiment Station, Bulletin series No. 334 (1942)

5. J.A. Sauer, A study of fatigue phenomena under combined stress, In: Proceedings of the seventh international congress for applied mechanics p. 150-164 (1948)

6. W.N. Findley. Combined-stress fatigue strength of 76S-T61 Aluminum alloy with superimposed mean stresses and corrections for yielding. NACA-TN-2924. NACA, Washington, (1953).

7. W.T. Chodorowski, Fatigue strength in shear of an alloy steel, with particular reference to the effect of mean stress and directional properties, In: Proc. Int. Conf. Fatigue of Metals. London: I. Mech. Eng. p. 122-131 (1956)

8. E. Haibach. FKM-Guideline: Analytical strength assessment of components in mechanical engineering (5th Rev Ed.), English version. Germany, Frankfurt/Main: Forschungskuratorium Maschinenebau (2003)

9. W.N. Findley. Effects of extremes of hardness and mean stress on fatigue of AISI 4340 steel in bending and torsion. Journal of Engineering Materials and Technology, 111: 119-122 (1989).

10. H. Mayer. Cyclic torsion very high cycle fatigue of VDSiCr spring steel at different load ratios. International Journal of Fatigue, 70: 322-327 (2015).

11. P. Davoli; A. Bernasconi; M. Filippini; S. Foletti; I.V. Papadopoulos. Independence of the torsional fatigue limit upon a mean shear stress. Int. J. Fatigue 25: 471-480 (2003)

12. C. Froustey, S. Laserre. Multiaxial fatigue endurance of 30 NCD16 steel. Int J Fatigue 11: $169-175$ (1989)

13. ASTM E466-15, Standard Practice for Conducting Force Controlled Constant Amplitude Axial Fatigue Tests of Metallic Materials. ASTM International, West Conshohocken, PA (2015)

14. W.N. Findley. A theory for the effect of mean stress on fatigue of metals under combined torsion and axial load or bending. J Eng. Ind. 81: 301-306 (1959)

15. J. Marin, Interpretation of fatigue strengths for combined stresses, In: Proceedings of international conference on fatigue of metals. London: Institution of Mechanical Engineers, p. 184-195 (1956)

16. C. Froustey, Contribution à l'étude de la fatigue multiaxiale sous sollicitations périodiques ou aléatoires, Thèse de Doctorat, Institut National des Sciences Appliquées de Lyon, Lyon (1992)

17. L. Pallarés-Santasmartas.; J. Albizuri; A. Avilés, A; R. Avilés. Mean Stress Effect on the Axial Fatigue Strength of DIN 34CrNiMo6 Quenched and Tempered Steel. Metals 8,213 (2018) 